2. Brody JP, Allen S, Schulman P, et al. Acute agranular CD4-positive natural killer cell leukemia. Comprehensive clinicopathologic studies including virologic and in vitro culture with inducing agents. Cancer 1995; 75: 2474-83.

3. Feuillard J, Jacob M-C, Valensi F, et al. Clinical and biologic features of CD4+/CD56+ malignancies. Blood 2002; 99: 1556-63.

4. Julia F, Dalle S, Duru G, et al. Blastic plasmacytoid dendritic cell neoplasms: clinico-immunohistochemical correlations in a series of $91 \mathrm{pa}-$ tients. Am J Surg Pathol 2014; 38: 673-80.

5. Pagano L, Valentini CG, Pulsoni A, et al. Blastic plasmacytoid dendritic cell neoplasm with leukemic presentation: an Italian multicenter study. Haematologica 2013; 98: 239-46.

6. Tsagarakis NJ, Kentrou NA, Papadimitriou KA, et al. Acute lymphoplasmacytoid dendritic cell (DC2) leukemia: results from the Hellenic Dendritic Cell Leukemia Study Group. Leuk Res 2010; 34: 438-46.

7. Rauh MJ, Rahman F, Good D, et al. Blastic plasmacytoid dendritic cel neoplasm with leukemic presentation, lacking cutaneous involvement: case series and literature review. Leuk Res 2012; 36: 81-6.

DOI: https://doi.org/10.1016/j.pathol.2018.05.007

\section{The delayed diagnosis of myeloproliferative neoplasms is common and results in a high incidence of potentially preventable thrombotic complications}

Sir,

There is a high incidence of thrombotic complications in patients with myeloproliferative neoplasms (MPN) prior to or at the time of diagnosis which reduces their quality of life and increases their risk of recurrent thrombotic events. We performed a retrospective chart review to determine the duration of blood count abnormalities prior to diagnosis and the incidence of potentially preventable thrombotic and haemorrhagic complications occurring during this time. In 147 patients included in this audit we found a significant delay from the appearance of MPN-associated blood count abnormalities until the diagnosis of a MPN was established. During this time $25 \%$ of the patients had a potentially preventable thrombotic or haemorrhagic event.

The Philadelphia $(\mathrm{Ph})$-negative myeloproliferative neoplasms (MPN) are a heterogeneous group of bone marrow disorders characterised by haematopoietic cellular proliferation with the excess production of mature blood cells resulting in characteristic anomalies in the peripheral blood.
Patients with these disorders are at increased risk of thrombotic and haemorrhagic complications, which contribute to morbidity and mortality, have an adverse impact on quality of life and are predictive of future thrombotic events. ${ }^{1,2}$ Thromboembolic events are reported to have occurred at or prior to diagnosis in $12-39 \%$ of polycythaemia vera (PV), 11-25\% of essential thrombocythaemia (ET) and $13.2 \%$ of primary myelofibrosis (PMF) patients. ${ }^{2,3}$ The frequency of thrombosis has been consistently reported to be greater than that of bleeding and those occurring at the time of diagnosis have traditionally been assumed to be unpreventable. We performed an audit of our MPN patients to determine the duration of blood count abnormalities prior to diagnosis and the incidence of potentially preventable thrombotic and haemorrhagic complications occurring during this time.

All adult patients diagnosed with a Ph-negative MPN [ET, PV, PMF or myeloproliferative neoplasm-unclassifiable (MPN-U)] between January 2010 and June 2017 under the care of haematologists at our centre were included in the audit. The blood count results of each patient were obtained and the date of the first appearance of a MPN-associated blood count abnormality was recorded. These included persistent polcythaemia, leukocytosis or thrombocytosis, or a leukoerythroblastic blood film. The hospital and specialist medical records of each patient were reviewed and the dates of any adverse thrombotic or haemorrhagic events potentially attributable to a MPN were identified. The time between the first blood count abnormality on record and diagnosis was recorded as diagnostic delay. All data were tabulated in a deidentified database and statistical analysis was performed.

The major results of the audit are summarised in Table 1. A total of 147 patients with a new diagnosis of a MPN were identified, including 37 patients with PV, 71 patients with ET, 26 patients with PMF and 13 patients with MPN-U.

The 37 patients with PV were all positive for JAK2V617F and had a mean age of 73 years (range $47-92$ years). The median diagnostic delay was 456 days (range $0-2650$ days). During this time 10 of the 37 patients (27\%) had potentially preventable complications: nine patients had thrombotic events which consisted of arterial events in seven patients (5 cerebrovascular, 2 cardiac events) and venous thrombosis in two patients, and one patient had a haemorrhagic event.

Of the 71 patients with ET, 63\% were positive for JAKV617F mutation, $14 \%$ were positive for a CALR mutation, $1.4 \%$ had a MPL mutation, $8.5 \%$ were triple-negative, and $12.7 \%$ were JAK2V617F negative but had no further

Table 1 Diagnosis delay and incidence of potentially preventable thrombotic and haemorrhagic complications

\begin{tabular}{|c|c|c|c|c|c|c|}
\hline \multirow[t]{3}{*}{ Diagnosis } & \multirow[t]{3}{*}{ No. patients } & \multirow[t]{3}{*}{ Median diagnosis delay, days (range) } & \multicolumn{4}{|c|}{ Number of patients with events } \\
\hline & & & \multirow[t]{2}{*}{ Total } & \multicolumn{2}{|c|}{ Thrombotic } & \multirow[t]{2}{*}{ Haemorrhagic } \\
\hline & & & & Arterial & Venous & \\
\hline Total & 142 & $723(0-8731)$ & $36(25 \%)$ & $22(15.5 \%)$ & $12(8.5 \%)$ & $4(2.8 \%)$ \\
\hline PV & 37 & $457(0-2650)$ & $10(27 \%)$ & $7(18.9 \%)$ & $2(5.5 \%)$ & $1(2.7 \%)$ \\
\hline ET & 71 & $825(0-8731)$ & $18(25 \%)$ & $13(18.3 \%)$ & $5(7 \%)$ & - \\
\hline MPN-U & 13 & $1371(442-3244)$ & $4(31 \%)$ & $1(7.7 \%)$ & $2(15.4 \%)$ & $3(23.1 \%)$ \\
\hline
\end{tabular}

ET, essential thrombocythaemia; MPN-U, myeloproliferative neoplasm-unclassifiable; PMF, primary myelofibrosis; PV, polycythaemia vera. 
testing. The mean age of this patient group was 66 years (range 20-89 years). The median diagnostic delay was 825 days (range $0-8731$ days). During this time 18 of the 71 patients $(25 \%)$ had potentially preventable thrombotic events: 13 patients had arterial events (including 6 cerebrovascular and 4 cardiac) and five had venous thrombosis. No patients were identified as having had a haemorrhagic event.

Of the 26 patients with PMF the mean age was 72 years (range 44-96 years). Mutation testing demonstrated that $54 \%$ were JAK2V617F positive, $8 \%$ were CALR positive, $4 \%$ were MPL positive, $8 \%$ were triple-negative and there was incomplete testing in $27 \%$. The median diagnostic delay was 257 days (range $0-3684$ days). During this time four of the 26 patients $(15 \%)$ had potentially preventable thrombotic events: one patient had a cardiac event and three had venous thrombosis. No patients were identified as having had a haemorrhagic event.

In the 13 patients with a MPN-U the mean age was 71 years (range 41-92 years). Mutation testing demonstrated that $77 \%$ were JAK2V617F positive, $8 \%$ were triple-negative and molecular testing was incomplete in the remainder. The median diagnostic delay was 1371 days (range 42-3255 days). Four patients $(31 \%)$ had potentially preventable events; three patients had thrombotic complications (2 venous thrombosis, 1 cerebrovascular event), and three had haemorrhagic events. Three patients had both haemorrhagic and thrombotic events.

We found a significant delay from the appearance of MPNassociated blood count abnormalities until the diagnosis of a MPN was established in our patient cohort. The longest delay was for patients with ET or MPN-U, whose MPN-associated blood count abnormality was characteristically a thrombocytosis which was present on average for almost 3 years prior to diagnosis. The diagnostic delay for patients with PV or PMF was significantly shorter at 18 months and 6 months, respectively, suggesting more understanding by the nonhaematologist of the clinical significance of polycythaemia and leukoerythroblastic blood films than of isolated thrombocytosis. During the time from first appearance of a blood count abnormality until diagnosis with a MPN, $25 \%$ of patients experienced a potentially preventable adverse event, predominantly thrombotic events. The prevalence of thrombotic complications in patients in our cohort diagnosed with MPNs is similar to that reported in literature at the time of diagnosis. $^{2,3}$

The number of haemorrhagic adverse events in our audit is likely to be under-reported as many events following minor surgical procedures in the community may not have been identifiable due to the retrospective nature of this chart review. Patients who had a fatal complication without a diagnosis of MPN were also not available to be included in this audit.

Factors which may contribute to delay in diagnosis are the lack of familiarity of general practitioners and nonhaematologist physicians with MPN-associated blood count abnormalities, as well as reporting laboratories not adequately emphasising the possibility of a diagnosis of MPN and not making recommendations for confirmation and further testing.

Thrombo-haemorrhagic events result in significant morbidity and mortality in patients with MPN. Improving awareness of blood count abnormalities suggestive of a MPN may facilitate the earlier diagnosis of a MPN, a reduction in the rate of complications at the time of diagnosis, an improved quality of life, and a reduction in the long-term thrombosis rate by earlier implementation of evidenced based treatment. ${ }^{4}$

The development and promulgation of guidelines for laboratory reporting of MPN-associated full blood count abnormalities may prompt the primary caregiver to consider the diagnosis of MPN in the presence of even mild peripheral blood abnormalities and initiate earlier diagnostic investigation and specialist referral.

Acknowledgement: This audit was approved by the Central Coast Local Health District Board Research Committee as a Quality Improvement activity (0216-013C). Colleagues who also contributed patients are Dr Tasman Armytage, Dr Brenton Wylie and Dr Mark Dean.

Conflicts of interest and sources of funding: The authors state that there are no conflicts of interest to disclose.

\section{Cecily Forsyth $^{1}$, Kathleen Melville ${ }^{2, *}$, Campbell Tiley ${ }^{2}$ \\ ${ }^{1}$ Wyong Hospital, Hamlyn Terrace, NSW, Australia; ${ }^{2}$ Gosford Hospital, Gosford, NSW, Australia; ${ }^{*}$ Current affiliation: Calvary Mater Newcastle, NSW, Australia}

Contact Dr Cecily Forsyth.

E-mail: cecily.forsyth@healthemail.com.au

1. Barbui T, Finazzi G, Falanga A. Myeloproliferative neoplasms and thrombosis. Blood 2013; 122: 2176-84.

2. Elliott MA, Tefferi A. Thrombosis and haemorrhage in polycythaemia vera and essential thrombocythaemia. Br J Haematol 2005; 128: 275-90.

3. Elliott MA, Pardanani A, Lasho TL, et al. Thrombosis in myelofibrosis: prior thrombosis is the only predictive factor and most venous events are provoked. Haematologica 2010; 95: 1788-91.

4. Mesa RA, Jamieson C, Bhatia R, et al. NCCN guidelines insights: myeloproliferative neoplasms, version 2.2018. J Natl Compr Cancer Netw 2017; 15: 1193-207.

DOI: https://doi.org/10.1016/j.pathol.2018.05.010

\section{Thrombotic thrombocytopenic purpura associated with Hodgkin lymphoma and non-Hodgkin lymphoma}

Sir,

Thrombotic thrombocytopenic purpura (TTP) is a rare haematological disease characterised by thrombotic microangiopathy in the setting of diminished ADAMTS13 enzyme activity. Presenting as a classic constellation of clinical and laboratory findings, it can be classified as primary, or secondary from autoimmune conditions, or malignancy. We present the first case of TTP secondary to Hodgkin lymphoma and low grade B cell lymphoma.

A 77-year-old female presented to the emergency department following 4 weeks of increased fatigue, weight loss, and pruritic rash. Past medical history was relevant for a recent total hip replacement, and hypertension. Initial blood tests revealed a mild normocytic anaemia, and normal platelets. Computed tomography (CT) scans showed extensive 